\title{
Treatment strategies that effectively reduce early recurrence risk in postmenopausal women with endocrine-sensitive breast cancer: AIs upfront vs. switching
}

\author{
Stefan Paepke $\cdot$ Volker R. Jacobs $\cdot$ Ralf Ohlinger • \\ Mathias Warm · Sherko Kümmel • Anke Thomas • \\ Nadia Harbeck • Marion Kiechle-Bahat
}

Received: 1 March 2007 / Accepted: 27 July 2007 / Published online: 6 September 2007

(C) Springer-Verlag 2007

\begin{abstract}
Several large, well-controlled clinical trials have now established that the aromatase inhibitors (AIs), including letrozole, anastrozole, and exemestane, are more effective than tamoxifen when used as adjuvant endocrine therapy in postmenopausal women with breast cancer. Yet, it is an open question as to how these drugs should be best integrated into the adjuvant treatment regimen. Both letrozole and anastrozole have shown efficacy over tamoxifen when used as initial adjuvant therapy (initiated just following surgery for breast cancer), while exemestane and anastrozole have been used as switching adjuvant therapy, i.e., following 2-3 years of initial adjuvant tamoxifen therapy, with proven efficacy over continued tamoxifen. Studies demonstrate that recurrence risk peaks in the early period after surgery, and that distant metastases in particular,
\end{abstract}

S. Paepke $(\bowtie) \cdot$ V. R. Jacobs $\cdot$ N. Harbeck $\cdot$ M. Kiechle-Bahat Interdisciplinary Breast Center, Operative Senology,

Frauenklinik (OB/GYN), Technical University Munich,

Ismaninger Strasse 22, 81675 Munich, Germany

e-mail: stefan.paepke@1rz.tum.de

\section{R. Ohlinger}

Interdisciplinary Breast Center, Frauenklinik,

Ernst-Moritz-Arndt-University, Greifswald, Germany

M. Warm

Interdisciplinary Breast Center, Frauenklinik,

University of Cologne, Cologne, Germany

\section{S. Kümmel}

Interdisciplinary Breast Center,

Department of Gynecology and Obstetrics,

University of Duisburg-Essen,

Duisburg-Essen, Germany

\section{A. Thomas}

Brustzentrum der Charité,

Humboldt University, Berlin, Germany accounting for most of the early recurrences, have worse survival rates when compared with other types of recurrences. Treatments that reduce recurrences, especially distant metastases, in this early period are therefore likely to improve overall survival (OS) and reduce mortality from breast cancer. In this review, we discuss early recurrence risk among postmenopausal women with successfully treated early breast cancer, the efficacy of the different AIs in reducing early recurrences and distant metastases when incorporated into adjuvant therapy, and the evidence for increased OS when AIs are used as initial or switch adjuvant therapy.

Keywords Adjuvant therapy - Anastrozole $\cdot$ Early breast cancer $\cdot$ Exemestane $\cdot$ Letrozole $\cdot$ Distant recurrence .

Tamoxifen

\section{Introduction}

There has been a sharp decrease in the incidence of breast cancer in the United States, which has been associated with the decreased use of hormone replacement therapy; however, cancer statistics continue to identify breast cancer as the single most commonly diagnosed cancer in women worldwide (Ravdin et al. 2006; Boyle and Ferlay 2005; Parkin et al. 1999) and a leading cause of cancer deaths among women, with nearly one million new cases diagnosed each year and about 373,000 cancer-related deaths yearly (Guarneri and Conte 2004). In Europe, breast cancer accounts for more than $25 \%$ of all newly diagnosed cancers and nearly $18 \%$ of all cancer-related deaths (Tyczynski et al. 2004). Early beliefs from the late 1800s that radical mastectomy-treated patients could be largely cured of breast cancer were proven wrong when it was found that 
only about $12 \%$ of these patients survived for 10 years after the procedure (Cianfrocca and Goldstein 2004). In fact, relapse most commonly occurs in the form of distant metastases, even in 20-30\% of node-negative (N0) patients (Cianfrocca and Goldstein 2004). The risk of distant metastases is much greater than the risk of locoregional recurrences, especially during the first few years post surgery (Mansell et al. 2006), and a distant metastatic event is particularly devastating for patients because at this stage, the cancer stops being curable and is associated with decreased survival (Lamerato et al. 2005).

Adjuvant chemotherapy and/or endocrine therapy is now the recommended norm to minimize the risk of recurrence among patients whose breast cancer has been diagnosed and surgically treated. Adjuvant therapy is not without risks, however, and patients wishing to reduce their recurrence risk must balance this benefit with any potential adverse events (AEs) associated with the adjuvant treatment. Assessing recurrence risk and defining which patients should undergo adjuvant therapy is therefore critical in the design of effective interventions.

The risk of recurrence can be substantial, especially in those patients with evidence of tumor dissemination to local lymph nodes; those with large or high grade tumors; those with low or no expression of hormonal receptor, including estrogen receptor (ER) in their tumors; and those whose tumors express known adverse prognostic indicators, such as the proto-oncogene c-erbB-2 (human epidermal growth factor receptor 2/neu) (Cianfrocca and Goldstein 2006). Although any type of disease recurrence is detrimental to the subsequent survival of breast cancer patients, distant metastases are a particularly ominous event, as this indicates the progression of disease to a largely incurable and ultimately fatal stage. Thus, while the goal of any adjuvant therapy is to prevent disease recurrence overall, those treatments that prove to prevent or significantly reduce distant metastases are likely to provide the most benefit for patients in terms of overall survival (OS) (Lamerato et al. 2005; Pantel and Brakenhoff 2004).

Adjuvant endocrine therapy is the standard treatment for postmenopausal women with breast cancer, as most breast cancers depend on estrogen for growth, and 5 years' treatment with tamoxifen, a selective ER modulator, has been the standard of care for many years (EBCTCG 2005). However, the aromatase inhibitors (AIs) have been approved in this indication and have displaced tamoxifen as the standard adjuvant treatment for postmenopausal women with endocrine-sensitive breast cancer. AIs act to inhibit the conversion of peripheral androgens to estrogen in postmenopausal women, effectively reducing plasma estrogen to near-undetectable levels (Choueiri et al. 2004; Dixon et al. 2006). The AIs anastrozole, letrozole, and exemestane have been introduced for the adjuvant treatment of breast cancer over the latter part of the past decade, and their use has been approved in selected treatment settings. With this addition of the AIs to the adjuvant treatment armamentarium, there is considerable debate over the most effective endocrine treatment strategy for postmenopausal women who have undergone successful surgery for early breast cancer. Results of the Arimidex, Tamoxifen, Alone or in Combination (ATAC) and Breast International Group (BIG 1-98) clinical trials suggest that anastrozole and letrozole are, respectively, more effective than tamoxifen in reducing recurrences when used as initial adjuvant treatment (Baum et al. 2002, 2003; Howell et al. 2005; Thurlimann et al. 2005). Other results from the Intergroup Exemestane Study (IES), the Italian-Tamoxifen-Arimidex (ITA) study, the Austrian Breast Cancer Study Group (ABCSG)-8 trial, and the Arimidex-Nolvadex (ARNO)-95 trial suggest that switching to exemestane or anastrozole following between 2 and 3 years of tamoxifen therapy is more effective than continuing tamoxifen out to 5 years (Coombes et al. 2004, 2007; Boccardo et al. 2005a; Jakesz et al. 2005a).

As relapses occur frequently during the first 5 years after diagnosis (Saphner et al. 1996), this time period is critical to preventing distant metastases and breast cancer-related mortality among patients. It is currently unclear which of these early adjuvant treatment strategies, if any, is superior to the others in the management of early breast cancer, or whether a particular strategy benefits any specific subgroup of patients. In this review, we discuss the risk of early breast cancer recurrence and the efficacy of different adjuvant treatment strategies involving AIs and tamoxifen in reducing this risk, to help physicians choose the treatment regimen that provides the most effective recurrence prophylaxis for their patients.

\section{Early recurrence risk}

A sizable proportion of breast cancer recurrences may occur in the early stages of adjuvant therapy. One study of more than 3,500 women with breast cancer undergoing adjuvant therapy (median follow-up 8.1 years) suggests that the greatest hazard for breast cancer recurrence occurs during the first few years after primary surgery; this was true for all degrees of nodal status, all sizes of primary tumors, and among both menopausal and postmenopausal patients (Saphner et al. 1996). Another more recently published investigation of more than 2,500 patients, 456 of whom had local, regional, or distant relapse, found that $79 \%$ of all recurrences occurred within the first 5 years, and well over half $(58 \%)$ occurred within the first 3 years following surgery (Elder et al. 2006). The site of recurrence also had a significant impact on disease-specific survival in this cohort; patients with a local recurrence had significantly 
better disease-specific survival than did those with recurrence at regional lymph nodes or distant metastases (Fig. 1) (Elder et al. 2006). Similar findings were reported in a retrospective cohort study of US patients $(N=1,616)$ with early breast cancer (Lamerato et al. 2005). In this study, the risk of dying was found to be over three times greater for patients with distant metastases as compared with a locoregional or contralateral recurrence (Fig. 2). These results suggest that while any recurrence of breast cancer is detrimental to long-term outcome, early distant metastases have the greatest impact; median survival following an isolated local recurrence has been reported to be 12.9 years, whereas the corresponding survival following a first distant metastasis event is only 2.2 years (Lê et al. 2002).

Predictors for early relapse (within 2.5 years from diagnosis) are similar to those for adverse breast cancer prognosis overall, and the results of three studies are summarized in Table 1. The collective results of these analyses suggest that factors such as large tumor size, grade III pathology, low ER positivity, and increasing lymph node involvement

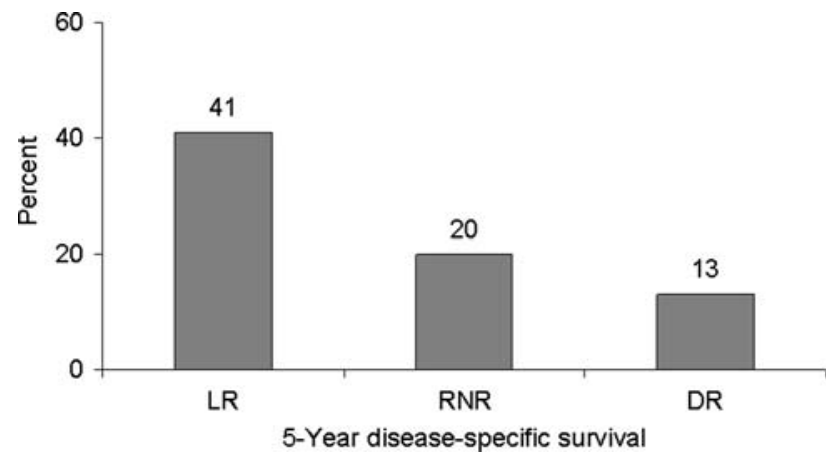

Fig. 1 5-Year disease-specific survival of breast cancer patients having local recurrence $(L R)$, recurrence at regional lymph nodes $(R N R)$, or distant recurrence $(D R)$. Disease-specific survival for LR was significantly different from RNR or DR patients $(P<0.0001)$ (Data from Elder et al. 2006)

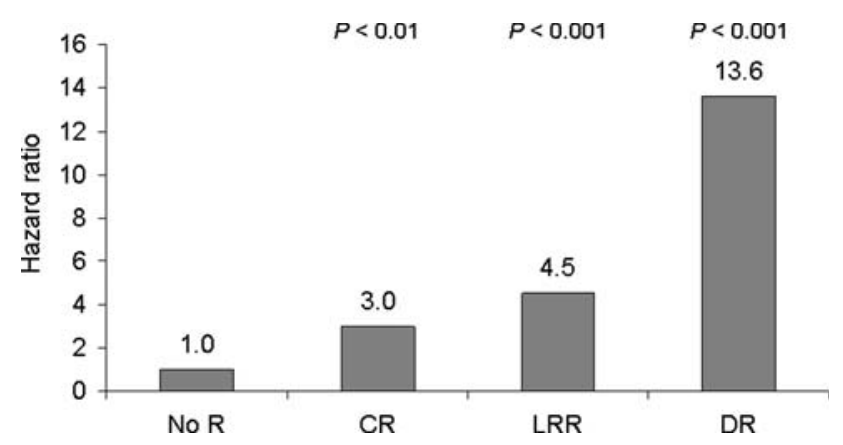

Fig. 2 Hazard ratio for death in patients with no recurrence (No $R$ ), contralateral recurrence $(C R)$, locoregional recurrence $(L R R)$, or distant recurrence $(D R)$. The associated $P$-value for comparison with No $\mathrm{R}$ group is shown (Data from Lamerato et al. 2005) are important to identify patients who are at the greatest risk for early recurrence, and who therefore should be treated aggressively with the most effective adjuvant therapy from the start (McArthur et al. 2005; Mansell et al. 2006; Mauriac et al. 2007). Yet, even for small tumors in the lowest risk category (N0, G1 tumors $<1 \mathrm{~cm}$ ), the 10 year risk of breast cancer recurrence is unexpectedly high, at $12 \%$ in the absence of adjuvant therapy (Chia et al. 2004).

It has been found that distant metastases comprise the majority of recurrence events that occur early in the course of breast cancer (Mansell et al. 2006). One study demonstrated that distant metastases occurred in 150 of 309 patients (49\%), and that 77 of these 150 distant metastases (51\%) occurred during the first 18 months of follow-up (Kryj et al. 1997). In multivariate analysis, the number of involved axillary lymph node and extracapsular invasion were found to be the most significant and independent factors involved in the development of distant metastases $(P<0.0001)$ (Kryj et al. 1997). Similar findings of an association between distant metastases and increasing lymph node positivity were reported in a study of breast cancers detected by screening or symptoms in the West Midlands, United Kingdom (Lawrence et al. 2005). However, the risk of developing an early distant metastasis is also present in the N0 patient population. One study estimated first distant metastases to occur at a rate of approximately $1-2 \%$ per month, $2-4 \%$ per month, and $3-6 \%$ per month in $\mathrm{T} 1$ patients (tumor diameter $1-7 \mathrm{~cm}$ ) who were N0, had 1-3 positive nodes, or had $>3$ positive nodes, respectively (Thames et al. 1999). Another study found the rate of early distant metastases to be between 6 and $8 \%$ in the N0 patients (Kryj et al. 1997). Other less significant predictors of distant metastases were tumor size and stage $(P<0.001)$, age $(P<0.003)$, and hormone responsiveness $(P<0.04)$ (Kryj et al. 1997).

Collectively, these studies indicate that as both N0 and node-positive $(\mathrm{N}+)$ patients, especially those with increasing tumor size and grade, are at substantive risk for early recurrences, and as the development of early distant metastases increases the risk of dying from the disease, these patients are candidates for early aggressive adjuvant therapy.

\section{Adjuvant tamoxifen treatment}

Given the adverse impact on OS associated with recurrence and especially early distant metastases, as outlined above, those adjuvant therapies that are proven to reduce the risk of early distant metastases are also the most likely to have a significant beneficial effect on OS. In the case of about 5 years of therapy with tamoxifen vs. not (in ER+ breast 
Table 1 Predictive factors for early disease recurrence (within 2.5 years) in studies of breast cancer patients (McArthur et al. 2005; Mansell et al. 2006; Mauriac et al. 2007)

\begin{tabular}{|c|c|c|c|}
\hline \multirow[t]{2}{*}{ Predictive factors } & \multicolumn{3}{|l|}{ Studies $N$ (follow-up) } \\
\hline & McArthur et al. 4,159 (7.4 years) & Mansell et al. 5,589 (60 months) & Mauriac et al. 5,980 (24 months) \\
\hline Age & Not predictive & Increasing age $(P=0.003)^{\mathrm{a}}$ & NR \\
\hline Tumor size & Not predictive & Increasing tumor size $(P=0.003)^{\mathrm{a}}$ & Large tumor size $(P=0.001)$ \\
\hline Tumor grade & Increasing grade $(P<0.001)$ & Increasing grade $(P<0.01)$ & Increasing grade $(P<0.001)$ \\
\hline Nodal involvement & $\begin{array}{l}\text { Increasing lymph node } \\
\quad \text { involvement }(P<0.001)\end{array}$ & $\begin{array}{l}\text { Increasing lymph node } \\
\text { involvement }(P<0.01)\end{array}$ & $\begin{array}{l}\text { Node-positive vs. node-negative } \\
\quad(P<0.001)\end{array}$ \\
\hline $\begin{array}{l}\text { Estrogen receptor } \\
\text { (ER) status }\end{array}$ & $\begin{array}{l}\text { ER expression moderate/high } \\
\quad \text { vs. low }(P=0.005)\end{array}$ & $\begin{array}{l}\text { Unknown ER status vs. } \\
\qquad \mathrm{ER}+(P=0.03)^{\mathrm{a}}\end{array}$ & $\begin{array}{l}\text { Absence of both ER/progesterone } \\
\text { receptor being positive }(P<0.001)\end{array}$ \\
\hline
\end{tabular}

cancer patients), results of the Oxford meta-analysis have demonstrated significant reductions in both disease recurrence $(41 \%)$ and breast cancer specific mortality (34\%). However, while most of the divergence between treatments in disease recurrence occurs during the first 5 years on treatment, the effect on breast cancer specific mortality is not observed until the period between 5 and 15 years (EBCTCG 2005). These findings are consistent with the notion that early reductions in recurrence can ultimately lead to improved survival for breast cancer patients, and argue for the early use of adjuvant therapy as a means to improve survival. In one study of 402 patients (median follow-up 50 months), allocation to adjuvant chemotherapy (88.8\% of patients), tamoxifen after chemotherapy (38\% of patients), or tamoxifen alone (11.2\% of patients) within 44 days after surgery was found to be associated with significantly improved OS $(P<0.05)$ but not disease-free survival (DFS) (Durnali et al. 2006).

Despite the benefit of large patient numbers and extended follow-up for many studies, and the time-proven benefits in terms of preventing recurrences and breast cancer specific mortality (EBCTCG 2005, 2006), there are a number of drawbacks to the use of tamoxifen as an adjuvant therapy for early breast cancer patients. The use of tamoxifen has been associated with serious complications including stroke, thromboembolism, and endometrial cancer. In the National Surgical Adjuvant Breast and Bowel Project (NSABP) P1 study examining tamoxifen relative to placebo in women at increased risk of breast cancer, patients allocated to tamoxifen had a 2.5-fold increased risk of invasive endometrial cancer, which occurred predominantly in women older than 50 years, a 1.6-fold increased risk of stroke, a 1.14-fold increased risk of developing cataracts, a one- to six-fold increased risk of deep vein thrombosis, and threefold increased risk of pulmonary embolus, the latter two events again occurring mainly in women 50 and older (Fisher et al. 1998). Other studies have confirmed the increased risk of thromboembolic events (pulmonary embolus and deep vein thrombosis), endometrial cancer, and stroke (McDonald et al. 1995; Braithwaite et al. 2003). In addition, gynecological events including hot flashes were reported to be "quite a bit or extremely" bothersome in nearly half of the patients on tamoxifen (45.7\%), and vaginal discharge that was "moderately bothersome or worse" occurred in about a third of the patients on tamoxifen (29\%) in the NSABP P1 trial (Fisher et al. 1998). These events may limit the tolerability of adjuvant tamoxifen, depending upon the individual patient. Among older patients (55 and older), the occurrence of side effects was associated with a fourfold higher rate of tamoxifen discontinuation; most troublesome among these events were depression, nausea, vision problems, and vaginal bleeding (Demissie et al. 2001). Tamoxifen is also associated with an excess of serious AEs (SAEs) such as endometrial cancer and venous thromboembolic events early on, in the first 2 years of treatment (Cuzick et al. 2006; Duffy 2006).

There is also some evidence from past clinical trials that tamoxifen is more effective in preventing locoregional recurrences as compared with distant metastases (Ludwig 1984; NATO 1988; Rutqvist et al. 1987). Given the detrimental effect of distant metastases as compared with locoregional recurrences on 5-year survival (Fig. 1), it is important to consider the efficacy of adjuvant therapies in preventing distant metastases at the earliest possible time point. Results from the Oxford meta-analysis also indicate an approximately $15 \%$ overall relapse rate within 5 years for patients allocated to about 5 years of tamoxifen, with an approximately $8 \%$ incidence of breast cancer specific mortality over the same time interval (EBCTCG 2005). Certain patient subgroups, including those with polymorphisms in the tamoxifen metabolizing enzyme cytochrome P D26, have also been shown to be more likely than others to relapse while on tamoxifen (wt/ $* 4$ or $* 4 / * 4$ genotype vs. wt $/ \mathrm{wt} ; 50 \%$ vs. $20.4 \% ; P=0.042)$, and such patients will 
therefore derive less benefit from the treatment (GonzalesSantiago et al. 2006). Clearly, there is evidence that a select group of patients is likely resistant to tamoxifen from the outset and would benefit from a more effective initial adjuvant therapy. We review below the clinical evidence that AIs are more effective than tamoxifen when used in the initial adjuvant setting (i.e., monotherapy with an AI or tamoxifen immediately following surgery for 5 years) or in the switch adjuvant setting (i.e., a switch to an AI following completion of 2 to 3 years of tamoxifen).

\section{Trials of initial adjuvant therapy with AIs}

\section{ATAC trial}

The ATAC trial was originally designed as a three-arm study comparing anastrozole, tamoxifen, or the combination as initial adjuvant therapy. However, as the initial analysis (33.3 months' follow-up) failed to demonstrate any benefit of the combination therapy arm over tamoxifen alone, this arm was subsequently discontinued (Baum et al. 2002, 2003). The most recent available analysis of this trial, obtained at a median of 68 months' follow-up (Howell et al. 2005), indicates a significant benefit of anastrozole over tamoxifen in DFS and time to distant metastases (TTDM) in the overall intent-to-treat population (Table 2), with a similar improvement in DFS [hazard ratio $(\mathrm{HR})=0.83 ; P=0.005]$ observed in the subgroup of hormone receptor-positive (HR+) patients. Of note, TTDM was not significantly improved in the $\mathrm{HR}+$ population (HR $=0.84 ; P=0.06)$. OS was thus similar for anastrozole vs. tamoxifen (Table 2), and the benefit of anastrozole in breast cancer specific mortality did not reach significance in the overall population (Table 2) (Howell et al. 2005). Of note, a recent analysis of the ATAC data estimated that the use of anastrozole in lieu of tamoxifen as initial adjuvant therapy would prevent 40,17 , and 14 recurrences, distant metastases, and deaths following recurrence, respectively, at 2.5 years and 82,44 , and 28 of these events, respectively, at 5 years (Houghton 2005).These data suggest that at 2.5 and 5 years of therapy, anastrozole was associated with fewer first events, recurrences, and deaths than was tamoxifen; most of the early impact of anastrozole was on locoregional and contralateral breast cancer recurrences rather than on distant metastases (Houghton 2006). In terms of safety, there was no difference in the overall incidence of AEs between the arms (94\% vs. 95\%, respectively), although there were significantly fewer treatment-related AEs $(61 \%$ vs. $68 \% ; P<0.0001)$, AEs leading to withdrawal ( $11 \%$ vs. $14 \%, P=0.0002)$, treatment-related AEs leading to withdrawal (6\% vs. $9 \% ; P=0.0005)$, SAEs (33\% vs. $36 \%, P=0.03$ ), treatment-related SAEs ( $5 \%$ vs.
9\%; $P<0.0001$ ), and SAEs leading to withdrawal (5\% vs. $6 \% ; P=0.04)$ in the anastrozole arm (ATAC 2006). When compared with tamoxifen, there was a significantly higher incidence of fractures $(11 \%$ vs. $8 \% ; P<0.0001)$ but not in patients with normal bone density at baseline. Incidences of osteopenia and osteoporosis $(11 \%$ vs. $7 \% ; P<0.0001)$ and hypercholesterolemia $(9.0 \%$ vs. $3.0 \% ; P<0.0001)$ were also significantly higher in the anastrozole arm (ATAC 2006). More ischemic cardiovascular events occurred in the anastrozole arm than in the tamoxifen arm, but the difference was not significant ( $4.1 \%$ anastrozole vs. $3.4 \%$ tamoxifen; $P=0.1)$. The incidences of endometrial cancer, thromboembolic events, vaginal bleeding, hot flashes, and vaginal discharge, on the other hand, all favored the use of anastrozole over tamoxifen (Howell et al. 2005).

\section{BIG 1-98 trial}

The BIG 1-98 trial was designed as a four-arm trial comparing an AI (letrozole) with tamoxifen as initial adjuvant therapy (either letrozole or tamoxifen for 5 years following surgery) or as sequential adjuvant therapy (letrozole for 2 years followed by tamoxifen for 3 years, or tamoxifen for 2 years followed by letrozole for 3 years) (Thurlimann et al. 2005). To increase its statistical power comparing upfront letrozole with tamoxifen, the first analysis included events occurring in the sequential arms with tamoxifen or letrozole as initial therapy. The results of this first primary core analysis ( $n=4,003$ letrozole, $n=4,007$ tamoxifen), at a median of 25.8 months' follow-up, have shown a significant benefit of initial adjuvant therapy with letrozole, with a $19 \%$ improvement in DFS ( $\mathrm{HR}=0.81 ; P=0.003$ ) over tamoxifen and a $27 \%$ reduction in the risk of distant metastases over tamoxifen (HR $=0.73 ; P=0.001)$. Results of the primary core analysis showed a total of 177 distant metastases events $(4.4 \%)$ in the letrozole arm compared with 232 $(5.8 \%)$ in the tamoxifen arm (Thurlimann et al. 2005). Protocol-specified subgroup analysis from BIG 1-98 also demonstrated a significant DFS benefit with letrozole over tamoxifen among those subgroups of patients who may be at increased risk for early distant metastases, including those with $\mathrm{N}+$ disease $(\mathrm{HR}=0.71 ; 95 \%$ CI $0.59-0.85$; $P<0.001)$, those with tumors $>2 \mathrm{~cm}(\mathrm{HR}=0.76 ; 95 \% \mathrm{CI}$ $0.63-0.92 ; P=0.004)$, and those receiving prior chemotherapy (HR $=0.70 ; 95 \%$ CI $0.54-0.92 ; P=0.01$ ) (Thurlimann et al. 2005).

The safety profile of letrozole vs. tamoxifen in this initial analysis showed a higher incidence of fractures $(5.7 \%$ vs. $4.0 \% ; P<0.001)$, arthralgia (20.3\% vs. $12.3 \% ; P<0.001)$, hypercholesterolemia $[5.4 \%$ vs. $1.2 \%$ (in patients who had normal baseline values that then became 1.5 times higher than the upper normal limits)], and cardiac failure $(0.8 \%$ vs. $0.4 \% ; P=0.01)$ in the letrozole group, while the tamoxifen 


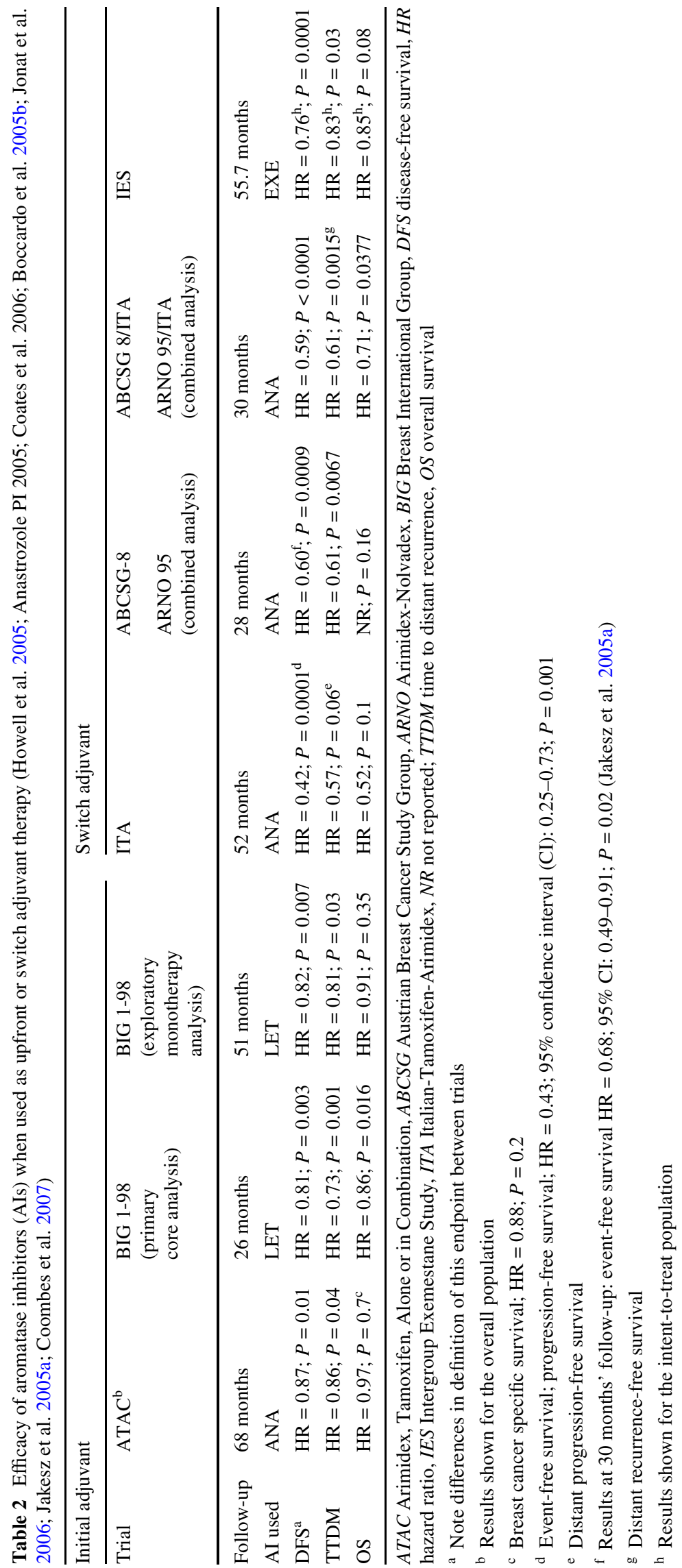


group showed significantly more thromboembolic events (3.5\% vs. $1.5 \% ; P<0.001)$, vaginal bleeding $(6.6 \%$ vs. $3.3 \% ; \quad P<0.001)$, hot flashes $(38.0 \%$ vs. $33.5 \%$; $P<0.001)$, and night sweats $(16.2 \%$ vs. $13.9 \% ; P=0.004)$ (Thurlimann et al. 2005; Letrozole 2005).

More recently, results from the exploratory analysis restricted to the two monotherapy arms, with a 51-month follow-up, have confirmed those findings ( $n=2,463$ letrozole and $n=2,459$ tamoxifen). The results continue to show a benefit of letrozole over tamoxifen, with an $18 \%$ improvement in DFS $(\mathrm{HR}=0.82 ; P=0.007)$ and a $19 \%$ significant reduction in the risk of distant metastases $(P=0.03)$ (Coates et al. 2007) (Table 2). The cumulative incidence of breast cancer relapse was $13.4 \%$ in the tamoxifen arm vs. $10.2 \%$ in the letrozole arm, a difference of $3.2 \%$ $(P=0.005)$. In addition, subgroup analysis continued to show the benefit of letrozole over tamoxifen in DFS in the $\mathrm{N}+$ group $(n=2,067 ; \mathrm{HR}=0.77 ; P=0.004)$, the chemotherapy-treated group $(n=1,232 ; \mathrm{HR}=0.74 ; P=0.03)$, and those with tumors $>2 \mathrm{~cm} \quad(n=1,858 ; \mathrm{HR}=0.79$; $P=0.01$ ) (Coates et al. 2007). An emerging benefit in the $\mathrm{N}-$ patient population as a $12 \%$ reduction in the risk of recurrence is also seen with longer follow-up ( $\mathrm{HR}=0.88$; $P=0.26$ ) (Coates et al. 2007). The safety results in this exploratory monotherapy arm analysis at 51 months were generally similar to those obtained in the initial analysis, with events such as vaginal bleeding, night sweats, and hot flushes more common in the tamoxifen arm, and bone fractures, arthralgia, low-grade hypercholesterolemia, and miscellaneous cardiovascular events observed more frequently in the letrozole group (Coates et al. 2007). Of note, thromboembolic events continued to be significantly reduced with letrozole vs. tamoxifen $(P<0.001)$, and the endpoints of any cardiac event, ischemic heart disease, and cardiac failure were not significantly different between the groups.

Results of the BIG 1-98 primary core analysis, obtained at a relatively early follow-up, as well as results from the exploratory monotherapy arm analysis, obtained at a longer follow-up, thus establish letrozole as a more effective alternative to tamoxifen in the initial adjuvant setting, and this effect appears to be especially pronounced on early distant metastases events. A recent analysis evaluating the predictors of early relapse in BIG 1-98 ( $N=5,890$ patients) demonstrated that distant metastases events constituted the majority of early events (within 2 years) (Mauriac et al. 2007); at this time, letrozole had already demonstrated a $30 \%$ reduction in the risk for distant metastases over tamoxifen (Thurlimann et al. 2005). This beneficial effect of letrozole in reducing early distant metastases is compelling given the adverse impact of distant metastases on survival, and should ultimately result in reduced breast cancer specific mortality, although this remains to be proven on longer follow-up.
Trials investigating AIs in the switch adjuvant setting

These trials have all generally investigated a strategy involving early treatment with tamoxifen for $2-3$ years, followed by an $\mathrm{AI}$ as adjuvant therapy for the remainder of the 5 -year treatment period. It is important to note that the defined switching period only includes those events starting from the switch point (after the end of tamoxifen therapy, at which time patients would either continue on tamoxifen or switch to an AI) until the study end.

\section{ITA trial}

This open-label trial compared the efficacy of a switch to anastrozole following 2-3 years of previous tamoxifen therapy with continued tamoxifen in 448 high-risk ER+ patients $(99 \% \mathrm{~N}+, 67 \%$ chemotherapy pretreatment) (Boccardo et al. 2005a, b). The switch from tamoxifen to anastrozole was associated with a significant reduction in breast cancer events (event-free survival and progression-free survival) and a $43 \%$ improvement in distant-progression-free survival at either the 36-month follow-up (HR $=0.49$; $95 \%$ CI $0.22-1.05 ; P=0.06$ ) or the 52-month follow-up (Table 2), with a marked reduction in locoregional recurrences at both of these follow-up points (36-month: $\mathrm{HR}=0.15 ; \quad P=0.003 ;$ 52-month: $\mathrm{HR}=0.13 ; P=0.002)$ (Boccardo et al. 2005b). More lipid disorders were seen with patients who switched to anastrozole $(9.3 \%$ vs. $4.0 \%$; $P=0.04$ ), while more gynecologic changes, including endometrial carcinoma, were observed in the patients on tamoxifen compared with anastrozole $(11.3 \%$ vs. $1.0 \%$; $P=0.0002)$, and significantly more SAEs were observed with tamoxifen ( $22 \%$ vs. $13.9 \% ; P=0.04$ ) (Boccardo et al. 2005a). Results of this trial thus suggest that switching to anastrozole after 2-3 years of tamoxifen results in fewer recurrences compared with continuing on tamoxifen.

\section{Combined ARNO 95/ABCSG 8 analysis}

The ARNO 95 and ABCSG 8 trials were also designed to evaluate the impact of a switch to anastrozole following 2 years of tamoxifen. An important difference in inclusion criteria, however, was that women were randomized pretreatment with tamoxifen in ABCSG 8 and after 2 years of tamoxifen in ARNO 95 (Jakesz et al. 2005a). The combined analysis examined events in 3,224 patients $(1,606$ continuing tamoxifen and 1,618 switching to anastrozole) at a median of 28 months following the initial treatment with tamoxifen (Jakesz et al. 2005a). Results demonstrated a $40 \%$ reduction in events with anastrozole vs. tamoxifen, and a $39 \%$ reduction in distant metastases (Table 2). Of note, distant metastases accounted for $62 \%$ of recurrences in women whose disease progressed, and this occurred in 
$3 \%$ of anastrozole-treated and 5\% of tamoxifen onlytreated patients; the HR for distant metastases as first events only was 0.54 in univariate analysis $(95 \%$ CI $0.37-0.80$; $P=0.0016$ ). By comparison, contralateral or ipsilateral recurrences accounted for only 16 and $28 \%$ of recurrences, respectively (Table 2). Results of this combined analysis thus suggest that switching to anastrozole after 2 years of tamoxifen is associated with reductions in distant breast cancer recurrences and recurrences overall, although no improvement in OS was observed. When considering only the ARNO 95 trial, however $(N=979)$, at 30.1 months of follow-up, Cox proportional hazards analysis demonstrated a 39\% improvement in DFS (38 events vs. 56 events; $\mathrm{HR}=0.66 ; 95 \% \mathrm{CI} 0.44-1.00 ; P=0.049$ ), as well as a $47 \%$ improvement in OS (15 anastrozole deaths vs. 28 tamoxifen deaths; HR, 0.53; 95\% CI 0.28-0.99; $P=0.045$ ) (Kaufmann et al. 2007).

\section{Combined analysis: ABCSG 8/ARNO 95/ ITA trials}

A further combined analysis of results from the ABCSG 8, ARNO 95, and ITA switching trials, which vary in trial design, patient populations, size, and doses of tamoxifen used, has been undertaken (Jonat et al. 2006). Results of this combined analysis of 4,006 patients $(2,009$ on anastrozole and 1,997 on tamoxifen), with a median follow-up of 30 months, demonstrated a significant $41 \%$ improvement in DFS, a $39 \%$ improvement in distant metastases-free survival, and a $29 \%$ improvement in OS with anastrozole compared with tamoxifen (Table 2). Patients switching to anastrozole experienced fewer recurrences (92 vs. 159) and deaths (66 vs. 90). The DFS benefit of switching to anastrozole vs. continuing on tamoxifen was also observed in patients with tumors $>2 \mathrm{~cm}(\mathrm{HR}=0.57)$, those with one to three positive nodes $(\mathrm{HR}=0.66)$, those with four or more positive nodes $(\mathrm{HR}=0.43)$, and those receiving prior chemotherapy $(\mathrm{HR}=0.33)$. These results thus suggest that the clinical benefit of anastrozole in terms of DFS ultimately translates into an OS benefit, and that physicians may consider switching patients currently taking adjuvant tamoxifen to an AI after 2-3 years.

\section{The IES}

The efficacy and safety of exemestane when used in the switch setting (following 2-3 years of tamoxifen) has also been examined in the IES. In this study, recurrence-free patients were randomized, after completing 2-3 years of tamoxifen, to either further tamoxifen or exemestane to complete 5 years of adjuvant therapy (Coombes et al. 2004). Initial results at a median follow-up of 30.6 months showed that there was a significant $32 \%$ benefit of exemestane over tamoxifen in DFS (HR $=0.68 ; P<0.001)$ and a significant $34 \%$ reduction in the risk of developing distant metastases $(\mathrm{HR}=0.66 ; P=0.0004)$, but only a trend toward improvement in $\mathrm{OS}(\mathrm{HR}=0.88 ; P=0.37)$.

Results of the IES at 55.7 months of follow-up demonstrated a significant $24 \%$ improvement in DFS, a significant $17 \%$ improvement in TTDM, and a non-significant $15 \%$ improvement in OS (Table 2). When only ER+ or unknown patients were included, the corresponding improvements in these endpoints was $25 \%(\mathrm{HR}=0.75 ; P=0.0001), 18 \%$ $(\mathrm{HR}=0.82 ; P=0.03)$, and $17 \%(\mathrm{HR}=0.83 ; P=0.05)$, with the latter OS endpoint being marginally significant (Coombes et al. 2007). After adjusting for ER+ status and pre-specified prognostic factors (e.g., nodal status and chemotherapy use), the difference in OS reached significance $(\mathrm{HR}=0.83 ; P=0.04)$. Results of the IES thus suggest that a switch to exemestane, like anastrozole, is more effective than continuing tamoxifen, and this benefit results in a survival advantage in ER+ patients. Moreover, they indicate that a significant reduction in the risk of distant metastases may predict a benefit in mortality related to breast cancer with longer follow-up.

AEs were similar to those seen in the initial analysis, with fewer thromboembolic events $(1.9 \%$ vs. $3.1 \% ; P=0.01)$, fewer serious gynecological AEs $(6.4 \%$ vs. 9.8\%; $P<0.001)$, and significantly more fractures $(7.0 \%$ vs. $4.9 \%$; $P=0.003)$ and arthralgia $(20.8 \%$ vs. $15.1 \% ; P<0.001)$ in the exemestane group compared with the tamoxifen group (Coombes et al. 2007). Also, patients who switched to exemestane showed an increased incidence of ischemic cardiac events $(9.9 \%$ vs. $8.6 \% ; P=0.12)$, angina $(7.1 \%$ vs. $6.5 \%$; $P=0.44)$, and myocardial infarction $(1.3 \%$ vs. $0.8 \%$; $P=0.08)$, although this was not statistically significant.

\section{Can we identify a superior AI or AI adjuvant strategy?}

The best adjuvant strategy is one that will allow for maximal long-term efficacy with good tolerability and a minimal risk of AEs that could lead to drug discontinuation. Efficacy is especially important in the early adjuvant setting, where the risk for breast cancer recurrence is greatest. Results from the ATAC and BIG 1-98 trials have demonstrated the superiority of AIs (anastrozole and letrozole, respectively) over tamoxifen in reducing recurrence risk in the initial adjuvant setting (Howell et al. 2005; Coates et al. 2007; Thurlimann et al. 2005) (Table 2). As detailed above, results from the IES, ABCSG 8, ARNO 95, and ITA trials also demonstrate that the switch to an AI (exemestane or anastrozole) after 2-3 years of adjuvant tamoxifen is more effective than continuing tamoxifen alone for 5 years, and a marginal survival benefit has also been demonstrated with this strategy (Coombes et al. 2007; Jonat et al. 2006) (Table 2). In view of these emerging data concerning the 
AIs, the American Society of Clinical Oncology Technology Assessment Team, the 2005 St Gallen consensus panel, and the National Comprehensive Cancer Network guidelines now include AIs as initial adjuvant therapy as an option for patients with endocrine-responsive disease (anastrozole and letrozole) and in the switch adjuvant setting (anastrozole and exemestane) (Goldhirsch et al. 2005; Carlson et al. 2006; Winer et al. 2005).

A comparison of risk reduction among the different AI trials might suggest on the surface that sequencing tamoxifen with an AI seems to provide a better relative risk reduction than the upfront use of an AI alone (e.g., compare initial and switch adjuvant trials; Table 2). Such comparisons, however, are not appropriate, because the patient populations selected in the switch setting differ from those in the initial adjuvant setting. It must be remembered that patients whose disease has recurred during the 2-3 years of tamoxifen therapy are excluded from the switching trials but not from the initial adjuvant trials (Pritchard 2005). It can also be argued that, by excluding these patients as well as patients who could not tolerate tamoxifen from the trial populations examined, one has already pre-selected a good prognosis patient population, one that has been shown to be responsive to endocrine therapy after 2-3 years of tamoxifen (Coombes et al. 2007; Boccardo et al. 2005a, Jakesz et al. 2005a; Kaufmann et al. 2006). Results of the switching trials have only demonstrated that there is a benefit in switching to AIs in patients disease-free after 2-3 years on tamoxifen.

In terms of tolerability, the side-effect profiles of the AIs differ from that of tamoxifen but appear to be generally similar across the three available drugs. The AIs thus offer benefits over tamoxifen in terms of risks for endometrial cancer and thromboembolic events at the expense of potential AEs on bone loss, and neutral effects on lipid profiles and cardiovascular health (Coombes et al. 2007; Boccardo et al. 2005a; Goss et al. 2005; Howell et al. 2005; Jakesz et al. 2005a; Thurlimann et al. 2005). There also appears to be no appreciable difference in terms of side effects when AIs are given as initial adjuvant therapy or as switch adjuvant therapy. Both initial and switch trials showed increased rates of gynecological events, including endometrial abnormalities, and thromboembolic events with tamoxifen compared with AIs, and increased rates of musculoskeletal events, including bone loss, with AIs compared with tamoxifen (Coombes et al. 2007; Boccardo et al. 2005a; Howell et al. 2005; Jakesz et al. 2005a; Thurlimann et al. 2005). Although both strategies also demonstrated increased rates of hypercholesterolemia and cardiac events with AIs compared with tamoxifen (Coombes et al. 2007; Boccardo et al. 2005a; Howell et al. 2005; Jakesz et al. 2005a; Thurlimann et al. 2005), these increases are not apparent in trials comparing AIs with placebo (Goss et al. 2005) and are likely explained by the cardioprotective effects that have been observed with tamoxifen (Braithwaite et al. 2003; EBCTCG 2005). In addition, neither treatment strategy has had a detrimental effect on patient quality of life in studies that have evaluated this endpoint (Fallowfield et al. 2004, 2006). While there are some arguments to support a prospectively planned sequencing strategy of tamoxifen with an $\mathrm{AI}$ in certain patients, i.e., those with low risk of recurrence, the only sequential trial that has reported results to date, ABCSG 8, has not yet shown any significant DFS benefit $(P=0.07)$ for the AI arm (tamoxifen followed by anastrozole vs. 5 years of initial adjuvant tamoxifen (Jakesz 2005b). Also, when considering the high rate of early recurrences, especially early distant metastases, the excess of SAEs, and withdrawals associated with adjuvant tamoxifen therapy in the first few years post surgery, the use of an $\mathrm{AI}$ over tamoxifen, in the time of this peak of recurrence risk, appears justifiable (Houghton 2005).

Additional data from the sequential arms of the BIG 198 trial are expected within the next 2 years. Once available, these should provide further information on the use of AIs (sequential vs. upfront) and any potential safety benefits of one approach vs. the other (Pritchard 2005). Until such data become available, it may be prudent to have all patients, and not just those at increased risk of relapse, begin their adjuvant therapy with the strongest available treatment in order to most effectively reduce the early relapse risk.

Several other unanswered questions remain regarding endocrine therapy. Studies to address the optimal duration of adjuvant AI therapy include MA.17R and NSABP B-42; both will examine whether there is an additional benefit with longer use of AIs (Goss 2006; Mamounas et al. 2006). In the MA.17R study, women who have successfully completed 5 years of extended adjuvant letrozole therapy (either in the MA.17 trial or routine clinical practice) will then be randomized to receive 5 more years of letrozole or placebo (Goss 2006). In the NSABP B-42 trial, the efficacy of 5 years of letrozole vs. placebo is being investigated in postmenopausal women with $\mathrm{HR}+$ breast cancer patients who have completed 5 years of endocrine therapy (Mamounas et al. 2006)

In addition, the Femara Reanalysed Through Genomics for Response Assessment, Calibration and Empowerment (FRAGRANCE) trial is investigating the genetic determinants responsible for de novo resistance to letrozole (ClinicalTrials.gov NCT00199134). Two other trials, Microarray In Node-Negative Disease May Avoid Chemotherapy (MINDACT) and Trial Assigning IndividuaLized Options for Treatment (TAILORx), are examining whether genetic testing can aid in the selection of the appropriate adjuvant treatment strategy, including the need for chemotherapy, 
for individual patients (ClinicalTrials.gov NCT00310180; ClinicalTrials.gov NCT00433589).

Which AI therapy, if any, is more effective and/or better tolerated than the others, whether used in the initial adjuvant or switch adjuvant setting, is not known. There are at present no results from large, well-controlled trials that directly compare the individual AIs with each other as adjuvant therapy in the same population. Upcoming results from at least two trials should provide more directly comparative efficacy and safety data for individual AIs. The MA.27 trial is comparing anastrozole with exemestane in the initial adjuvant setting (Pritchard 2005; ClinicalTrials.gov NCT00066573). The Femara vs. Anastrozole Clinical Evaluation (FACE) trial is evaluating the efficacy and safety of 5 years of adjuvant anastrozole vs. letrozole as initial adjuvant therapy with the increased-risk population of $\mathrm{N}+$ patients, so that the results may be obtained more quickly (DeBoer et al. 2006). Another trial with exemestane, the Tamoxifen and Exemestane Adjuvant Multicenter (TEAM) trial, was started in 2001 to compare exemestane or tamoxifen as adjuvant monotherapy for 5 years but was then amended, based on the results from IES, to investigate sequencing from tamoxifen to exemestane after 2 to 3 years in comparison with 5 years of exemestane (Markopoulos et al. 2006; ClinicalTrials.gov NCT00032136). Publication of efficacy results is still pending.

\section{Conclusions}

While the AIs have a proven superiority over tamoxifen when used as either initial or switch adjuvant therapy (Howell et al. 2005; Coombes et al. 2007; Thurlimann et al. 2005; Boccardo et al. 2005a; Jakesz et al. 2005a), the optimal AI and adjuvant strategy remains a matter of debate. The use of AIs rather than tamoxifen, with either strategy, is supported by good clinical data, and there appears to be little difference in safety with either approach. The upcoming results from the sequential arms of the BIG 1-98 trial may provide some data as to which strategy (AI upfront or used sequentially), if either, is most effective and/or is better tolerated. In the meantime, while awaiting these data and those from directly comparative AI studies such as FACE and MA.27, it is clear that patients with endocrinesensitive breast cancer remain at a substantial risk for recurrence, especially during the early years after surgery, and that there is a risk for early distant relapse, and consequently death from breast cancer, in women with $\mathrm{N}+$ and with $\mathrm{N}-$ disease. Distant metastases recurrence events are the most common type of early relapse event, and for postmenopausal patients with endocrine-sensitive breast cancer, beginning therapy with an AI offers the best opportunity to reduce the risk of early relapse. Of note, letrozole is partic- ularly effective at reducing the risk of distant metastases in patients with $\mathrm{HR}+$ tumors in the initial adjuvant setting. An $\mathrm{AI}$ should be used as early as possible in the course of treatment, as the increased risks of early recurrence observed with tamoxifen cannot be offset by the later use of an AI. On the other hand, for patients who did not have the chance to start with an AI upfront, using an AI sequentially after tamoxifen is a reasonable option and provides greater protection against relapse than continuing on tamoxifen.

\section{References}

Arimidex, Tamoxifen, Alone or in Combination Trialists' Group, Buzdar A, Howell A, Cuzick J, Wale C, Distler W, Hoctin-Boes G, Houghton J, Locker GY, Nabholtz JM (2006) Comprehensive side-effect profile of anastrozole and tamoxifen as adjuvant treatment for early-stage breast cancer: long-term safety analysis of the ATAC trial. Lancet Oncol 7:633-643

Baum M, Budzar AU, Cuzick J, Forbes J, Houghton JH, Klijn JG, Sahmoud T, ATAC Trialists' Group (2002) Anastrozole alone or in combination with tamoxifen versus tamoxifen alone for adjuvant treatment of postmenopausal women with early breast cancer: first results of the ATAC randomized trial. Lancet 359:21312139 (Erratum in: Lancet 360:1520)

Baum M, Buzdar A, Cuzick J, Forbes J, Houghton J, Howell A, Sahmoud T, the ATAC (Arimidex, Tamoxifen Alone or in Combination) Trialists' Group (2003) Anastrozole alone or in combination with tamoxifen versus tamoxifen alone for adjuvant treatment of postmenopausal women with early-stage breast cancer: results of the ATAC (Arimidex, Tamoxifen Alone or in Combination) trial efficacy and safety update analyses. Cancer 98:1802-1810

Boccardo F, Rubagotti A, Puntoni M, Guglielmini P, Amoroso D, Fini A, Paladini G, Mesiti M, Romeo D, Rinaldini M, Scali S, Porpiglia M, Benedetto C, Restuccia N, Buzzi F, Franchi R, Massidda B, Distante V, Amadori D, Sismondi P (2005a) Switching to anastrozole versus continued tamoxifen treatment of early breast cancer: preliminary results of the Italian Tamoxifen Anastrozole trial. J Clin Oncol 23:5138-5147

Boccardo FM, Rubagotti A, Puntoni M, Guglielmini P, Porpiglia M, Mesiti M, Rinaldini M, Paladini G, Distante V, Franchi R, ITA trialists (2005b) Switching to anastrozole (ANA) vs continued tamoxifen (TAM) treatment of early breast cancer (EBC). Updated results of the Italian Tamoxifen Anastrozole (ITA) trial. J Clin Oncol 25(16S): 10s (abstract 526)

Boyle P, Ferlay J (2005) Cancer incidence and mortality in Europe, 2004. Ann Oncol 16:481-488

Braithwaite RS, Chlebowski RT, Lau J, George S, Hess R, Col NF (2003) Meta-analysis of vascular and neoplastic events associated with tamoxifen. J Gen Intern Med 18:937-947

Carlson RW, Brown E, Burstein HJ, Gradishar WJ, Hudis CA, Loprinzi C, Mamounas EP, Perez EA, Pritchard K, Ravdin P, Recht A, Somlo G, Theriault RL, Winer EP, Wolff AC, National Comprehensive Cancer Network (2006) NCCN task force report: adjuvant therapy for breast cancer. J Natl Compr Canc Netw 4(Suppl 1):S1-S26

Chia SK, Speers CH, Bryce CJ, Hayes MM, Olivotto IA (2004) Tenyear outcomes in a population-based cohort of node-negative, lymphatic, and vascular invasion-negative early breast cancers without adjuvant systemic therapies. J Clin Oncol 22:1630-1637

Choueiri TK, Alemany CA, Abou-Jawde RM, Budd GT (2004) Role of aromatase inhibitors in the treatment of breast cancer. Clin Ther 26:1199-1214 
Cianfrocca M, Goldstein LJ (2004) Prognostic and predictive factors in early-stage breast cancer. Oncologist 9:606-616

ClinicalTrials.gov. Identifier: NCT00066573. www.clinicaltrials.gov/ $\mathrm{ct} /$ show/NCT00066573 order $=1$. accessed 14 February 2007

ClinicalTrials.gov. Identifier: NCT00199134. www.clinicaltrials.gov/ ct/show/NCT00199134? order $=5$. accessed 19 July 2007

ClinicalTrials.gov. Identifier: NCT00310180. www.clinicaltrials.gov/ ct/show/NCT00310180?order $=1$. accessed 19 July 2007

ClinicalTrials.gov. Identifier: NCT00032136. www.clinicaltrials.gov/ $\mathrm{ct} /$ show/NCT00032136? order $=1$. accessed 19 July 2007

ClinicalTrials.gov. Identifier: NCT00433589 www.clinicaltrials.gov/ $\mathrm{ct} /$ show/NCT00433589? order $=1$. accessed 19 July 2007

Coates AS, Keshaviah A, Thurlimann B, Mouridsen H, Mauriac L, Forbes JF, Paridaens R, Castiglione-Gertsch M, Gelber RD, Colleoni M, Lang I, Del Mastro L, Smith I, Chirgwin J, Nogaret JM, Pienkowski T, Wardley A, Jakobsen EH, Price KN, Goldhirsch A (2007) Five years of letrozole compared with tamoxifen as initial adjuvant therapy for postmenopausal women with endocrineresponsive early breast cancer: update of study BIG 1-98. J Clin Oncol 25:486-492

Coombes RC, Hall E, Gibson LJ, Paridaens R, Jassem J, Delozier T, Jones SE, Alvarez I, Bertelli G, Ortmann O, Coates AS, Bajetta E, Dodwell D, Coleman RE, Fallowfield LJ, Mickiewicz E, Andersen J, Lonning PE, Cocconi G, Stewart A, Stuart N, Snowdon CF, Carpentieri M, Massimini G, Bliss JM, van de Velde C, Intergroup Exemestane Study (2004) A randomized trial of exemestane after two to three years of tamoxifen therapy in postmenopausal women with primary breast cancer. $\mathrm{N}$ Engl J Med 350:1081-1092 (Erratum in: N Engl J Med 351:2461)

Coombes RC, Kilburn LS, Snowdon CF, Paridaens R, Coleman RE, Jones SE, Van de Velde CJH, Delozier T, Alvarez I, Del Mastro L, Ortmann O, Diedrich K, Coates AS, Bajetta E, Holmberg SB, Dodwell D, Mickiewecz E, Andersen J, Lønning PE, Cocconi G, Forbes J, Castiglione M, Stuart N, Stewart A, Fallowfield LJ, Bertelli G, Hall E, Bogle RG, Carpentieri M, Colajori E, Subar M, Ireland $\mathrm{E}$, Bliss $\mathrm{JM}$, on behalf of the Intergroup Exemestane Study (2007) Survival and safety of exemestane versus tamoxifen after 2-3 years' tamoxifen treatment (Intergroup Exemestane Study): a randomised controlled trial. Lancet 369:559-570 (Erratum in: Lancet 369:906)

Cuzick J, Wale C, on Behalf of the ATAC Trialists' Group (2006) A detailed analysis of the benefits of anastrozole over tamoxifen for venous thromboembolic events (VTEs) after 5 years' treatment. Breast Cancer Res Treat 100(Suppl 1):S24 (abstract 104)

De Boer R, Burris H, Monnier A, Mouridsen H, O'Shaughnessy J, McIntyre K, Pritchard K, Smith I, Yardley D, on behalf of the H2H trial steering committee The Head to Head trial (2006) Letrozole vs anastrozole as adjuvant treatment of postmenopausal patients with node positive breast cancer. J Clin Oncol 24(18S):582s (abstract 10672)

Demissie S, Silliman RA, Lash TL (2001) Adjuvant tamoxifen: predictors of use, side effects, and discontinuation in older women. J Clin Oncol 19:322-328

Dixon JM, Renshaw L, Young O, Murray J, Macaskill EJ, McHugh M, Folkerd E, Cameron D, Dowsett M (2006) Letrozole suppresses plasma oestradiol (E2) levels more completely than anastrozole in postmenopausal women with breast cancer. J Clin Oncol 24(18S):15s (abstract 552)

Duffy S, on Behalf of the ATAC Trialists' Group (2006) Anastrozole is associated with a lower risk of endometrial abnormalities than tamoxifen: first report of the ATAC trial endometrial sub-protocol at 6 years' follow-up. Breast Cancer Res Treat 100(Suppl 1):S190 (abstract 4055)

Durnalli AG, Alkis N, Utkan G, Celenkoglu G, Arslan UY, Muallaoglu S, Kocer M, Tokluoglu S (2006) What is the optima starting time of adjuvant treatment in breast cancer patients? Ann Oncol 17(Suppl 9):ix108 (abstract 301)

Early Breast Cancer Trialists' Collaborative Group (EBCTCG) (2005) Effects of chemotherapy and hormonal therapy for early breast cancer on recurrence and 15-year survival: an overview of the randomised trials. Lancet 365:1687-1717

EBCTCG Secretariat, on behalf of EBCTCG (2006) Highlights from the Early Breast Cancer Trialists' Collaborative Group (EBCTCG) worldwide overview. Breast Cancer Res Treat 100(Suppl 1):S19 (abstract 40)

Elder EE, Kennedy CW, Gluch L, Carmalt HL, Janu NC, Joseph MG, Donellan MJ, Molland JG, Gillett DJ (2006) Patterns of breast cancer relapse. Eur J Surg Oncol 32:922-927

Fallowfield L, Cella D, Cuzick J, Francis S, Locker G, Howell A (2004) Quality of life of postmenopausal women in the Arimidex, Tamoxifen, Alone or in Combination (ATAC) adjuvant breast cancer trial. J Clin Oncol 22:4261-4271

Fallowfield LJ, Bliss JM, Porter LS, Price MH, Snowdon CF, Jones SE, Coombes RC, Hall E (2006) Quality of life in the intergroup exemestane study: a randomized trial of exemestane versus continued tamoxifen after 2 to 3 years of tamoxifen in postmenopausal women with primary breast cancer. J Clin Oncol 24:910 917

Fisher B, Costantino JP, Wickerham DL, Redmond CK, Kavanah M, Cronin WM, Vogel V, Robidoux A, Dimitrov N, Atkins J, Daly M, Wieand S, Tan-Chiu E, Ford L, Wolmark N (1998) Tamoxifen for prevention of breast cancer: report of the National Surgical Adjuvant Breast and Bowel Project P-1 Study. J Natl Cancer Inst 90:1371-1388

Goldhirsch A, Glick JH, Gelber RD, Coates AS, Thurlimann B, Senn HJ, Panel Members (2005) Meeting highlights: international expert consensus on the primary therapy of early breast cancer 2005 . Ann Oncol 16:1569-1583

Gonzales-Santiago S, Zarate R, de la Haba-Rodriguez J, Gómez A, Bandres E, Borrega P, Garcia-Foncillas J, Aranda E (2006) Genetic polymorphism CYP2D6(*4) interaction in clinical outcomes of tamoxifen-treated breast cancer patients. Ann Oncol 17(Suppl 9):ix62 (abstract 114P)

Goss P (2006) Update on the MA.17 extended adjuvant trial. Best Pract Res Clin Endocrinol Metab 20(Suppl 1):S5-S13

Goss PE, Ingle JN, Martino S, Robert NJ, Muss HB, Piccart MJ (2005) Randomized trial of letrozole following tamoxifen as extended adjuvant therapy in receptor-positive breast cancer: updated findings from NCIC CTG MA.17. J Natl Cancer Inst 97:1262-1271

Guarneri V, Conte PF (2004) The curability of breast cancer and the treatment of advanced disease. Eur J Nucl Med Mol Imaging 31(Suppl 1):S149-S161

Houghton J, on behalf of the ATAC Trialists' Group (2005) Using anastrozole as initial adjuvant treatment prevents early recurrences and reduces adverse events: Updated data from the ATAC ('Arimidex', Tamoxifen, Alone or in Combination) trial. J Clin Oncol 23(16S):24S (abstract 582)

Houghton J, on behalf of the ATAC Trialists' Group (2006) Initial adjuvant therapy with ANA (S) reduced rates of early breast cancer recurrent and adverse events compared with TAM (T)—data reported on behalf of the ATAC ('Arimidex', Tamoxifen, Alone and in Combination) Trialists' group. Ann Oncol 17(Suppl 9):ix93 (abstract 243PD)

Howell A, Cuzick J, Baum M, ATAC Trialists' Group, et al (2005) Results of the ATAC (Arimidex, Tamoxifen, Alone or in Combination) trial after completion of 5 years' adjuvant treatment for breast cancer. Lancet 365:60-62

Jakesz R, Jonat W, Gnant M, Mittlboeck M, Greil R, Tausch C, Hilfrich J, Kwasny W, Menzel C, Samonigg H, Seifert M, Gademann G, Kaufmann M, Wolfgang J, ABCSG, the GABG (2005a) Switching of postmenopausal women with endocrine-responsive 
early breast cancer to anastrozole after 2 years' adjuvant tamoxifen: combined results of ABCSG trial 8 and ARNO 95 trial. Lancet 366:455-462

Jakesz R, Gnant M, Greil R, Tausch C, Samonigg H, Kwasny W, Kubista E, Stierer M, Luschin G, Mittlboeck M (2005b) The benefits of sequencing adjuvant tamoxifen and anastrozole in postmenopausal women with hormone-responsive early breast cancer: 5 year-analysis of ABCSG Trial 8. Breast Cancer Res Treat 94(Suppl 1):S10 (abstract 13)

Jonat W, Gnant M, Boccardo F, Kaufmann M, Rubagotti A, Zuna I, Greenwood M, Jakesz R (2006) Effectiveness of switching from adjuvant tamoxifen to anastrozole in postmenopausal women with hormone-sensitive early-stage breast cancer: a meta analysis. Lancet Oncol 7:991-996

Kaufmann M, Jonat W, Hilfrich J, Eidtmann H, Gademann G, Zuna I, von Minckwitz G (2007) Improved overall survival in postmenopausal women with early breast cancer after anastrozole initiated after treatment with tamoxifen compared with continued tamoxifen: the ARNO 95 study. J Clin Oncol 25:2664-2670

Kryj M, Maciejewski B, Withers HR, Taylor JM (1997) Incidence and kinetics of distant metastases in patients with operable breast cancer. Neoplasma 44:3-11

Lamerato L, Havstad S, Gandhi S, Jones D, Chlebowski R (2005) Breast cancer recurrence and related mortality in US pts with early breast cancer. J Clin Oncol 23(16S):62s (abstract 738)

Lawrence G, Pritchard MG, Kearins O, Casey M (2005) An analysis of breast cancer recurrences for screen detected and symptomatic breast cancers diagnosed in the West Midlands in 1996 and 1997. Breast Cancer Res Treat 94(Suppl 1):S211 (abstract 5019)

Lê MG, Arriagada R, Spielmann M, Guinebretiére J-M, Rochard F (2002) Prognostic factors for death after an isolated local recurrence in patients with early-stage breast carcinoma. Cancer 94:2813-2820

Ludwig Breast Cancer Study Group (1984) Randomised trial of chemo-endocrine therapy, endocrine therapy, and mastectomy alone in postmenopausal patients with operable breast cancer and axillary node metastasis. Lancet 1:1256-1260

Mamounas EP, Lembersky B, Jeong JH, Cronin W, Harkins B, Geyer C, Wickerham DL, Paik S, Costantino J, Wolmark N (2006) NSABP B-42: a clinical trial to determine the efficacy of five years of letrozole compared with placebo in patients completing five years of hormonal therapy consisting of an aromatase inhibitor (AI) or tamoxifen followed by an $\mathrm{AI}$ in prolonging disease-free survival in postmenopausal women with hormone receptor-positive breast cancer. Clin Breast Cancer 7:416-421

Mansell R, Monypenny IJ, Skene AI, Abram P, Gattuso J, Abdel-Rahman A, Wilson CR, Angerson WF, Doughty JC (2006) Predictors of early recurrence in postmenopausal women with operable breast cancer. Breast Cancer Res Treat 100(Suppl 1):S111 (abstract 2091)

Markopoulos C, Polychronis A, Zobolas V, Xepapadakis G, Papadiamantis J, Koukouras D, Lappas H, Gogas H (2006) The effect of exemestane on the lipidemic profile of postmenopausal early breast cancer patients: preliminary results of the TEAM Greek sub-study. Breast Cancer Res Treat 93:61-66

Mauriac L, Keshaviah A, Debled M, Mouridsen H, Forbes J, Thurlimann B, Paridaens R, Monnier A, Lang I, Wardley A, Nogaret JM, Gelber R, Castiglione-Gertsch M, Price K, Coates A, Smith I, Viale G, Rabaglio M, Zabaznyi N, Goldhirsch A (2007) Predic- tors of early relapse in postmenopausal women with hormone receptor-positive breast cancer in the BIG 1-98 trial. Ann Oncol 18:859-867

McArthur HL, Olivotto I, Gelmon KA, Speers CH, Chia S, Ellard S, Kennecke JF (2005) Risk of early relapse in post-menopausal women with early stage, estrogen receptor positive $(\mathrm{ER}+)$ breast cancer on tamoxifen. Breast Cancer Res Treat 94(Suppl 1):S124 (abstract 3001)

McDonald CC, Alexander FE, Whyte BW, Forrest AP, Stewart HJ (1995) Cardiac and vascular morbidity in women receiving adjuvant tamoxifen for breast cancer in a randomised trial. The Scottish Cancer Trials Breast Group. BMJ 311:977-980

'Nolvadex' Adjuvant Trial Organisation (1988) Controlled trial of tamoxifen as a single adjuvant agent in the management of early breast cancer. Br J Cancer 57:608-611

Novartis Pharmaceuticals Corporation (2005) Letrozole [package insert]. Novartis Pharmaceuticals Corporation, East Hanover

Pantel K, Brakenhoff RH (2004) Dissecting the metastatic cascade. Nat Rev Cancer 4:448-456

Parkin DM, Pisani P, Ferlay J (1999) Global cancer statistics. CA Cancer J Clin 49(1):33-64

Pritchard KI (2005) Aromatase inhibitors in adjuvant therapy of breast cancer: before, instead of, or beyond tamoxifen. J Clin Oncol 23:4850-4852

Ravdin PM, Cronin KA, Howlander N, Chlebowski RT, Berry DA (2006) A sharp decrease in breast cancer incidence in the United States in 2003. Breast Cancer Res Treat 100(Suppl 1):S6 (abstract 5)

Rutqvist LE, Cedermark B, Glas U, Johansson H, Nordenskjold B, Skoog L, Somell A, Theve T, Friberg S, Askergren J (1987) The Stockholm trial on adjuvant tamoxifen in early breast cancer. Correlation between estrogen receptor level and treatment effect. Breast Cancer Res Treat 10:255-266

Saphner T, Tormey DC, Gray R (1996) Annual hazard rates of recurrence for breast cancer after primary therapy. J Clin Oncol 14:2738-2746

Thames HD, Buchholz TA, Smith CD (1999) Frequency of first metastatic events in breast cancer: implications for sequencing of systemic and local-regional treatment. J Clin Oncol 17:2649-2658

Thurlimann B, Keshaviah A, Coates AS, Mouridsen H, Mauriac L, Forbes JF, Paridaens R, Castiglione-Gertsch M, Gelber RD, Rabaglio M, Smith I, Wardley A, Price KN, Goldhirsch A, Breast International Group (BIG) 1-98 Collaborative Group (2005) A comparison of letrozole and tamoxifen in postmenopausal women with early breast cancer. N Engl J Med 353:2747-2757

Tyczynski JE, Plesko I, Aareleid T, Primic-Zakelj M, Dalmas M, Kurtinaitis J, Stengrevics A, Parkin DM (2004) Breast cancer mortality patterns and time trends in 10 new EU member states: mortality declining in young women, but still increasing in the elderly. Int J Cancer 112:1056-1064

Winer EP, Hudis C, Burstein HJ, Wolff AC, Pritchard KI, Ingle JN, Chlebowski RT, Gelber R, Edge SB, Gralow J, Cobleigh MA, Mamounas EP, Goldstein LJ, Whelan TJ, Powles TJ, Bryant J, Perkins C, Perotti J, Braun S, Langer AS, Browman GP, Somerfield MR (2005) American Society of Clinical Oncology technology assessment on the use of aromatase inhibitors as adjuvant therapy for postmenopausal women with hormone receptor-positive breast cancer: status report 2004. J Clin Oncol 23:619-629 